\title{
Percepção dos estudantes do curso de ciências biológicas de uma universidade pública quanto à importância da disciplina de bioterismo e biossegurança
}

\author{
Perception of the students of the course of biological sciences of a public \\ university as to the importance of the discipline of biotherism and biosafety
}

DOI: 10.46814/lajdv3n3-021

Recebimento dos originais: 01/05/2021

Aceitação para publicação: 30/06/2021

\author{
Isla Vanessa Gomes Alves Bastos \\ Licenciatura em Ciências Biológicas \\ Universidade Federal Rural de Pernambuco (UFRPE) \\ Rua Dom Manuel de Medeiros, s/n, CEP: 52.171-900, Dois Irmãos, Recife /PE. \\ E-mail: bastosisla@gmail.com \\ Thiago Bruno Lima da Silva \\ Licenciatura em Ciências Biológicas \\ Universidade Federal Rural de Pernambuco - UFRPE \\ Rua Dom Manuel de Medeiros, s/n, CEP: 52.171-900, Dois Irmãos, Recife /PE. \\ E-mail: thiago.bls72@gmail.com \\ Anísio Francisco Soares \\ Doutor em Ciências Biológicas, Área de Concentração Fisiologia. \\ Departamento de Morfologia e Fisiologia Animal - DMFA \\ Programa de Pós-Graduação em Biociência Animal - PPGBA \\ Universidade Federal Rural de Pernambuco - UFRPE \\ Rua Dom Manuel de Medeiros, s/n, CEP: 52.171-900, Dois Irmãos, Recife /PE. \\ E-mail: anisiofsoares@gmail.com
}

\begin{abstract}
RESUMO
A contínua evolução do conhecimento humano, especialmente o da biologia, promove ações que envolvem a criação e a experimentação animal, proporcionando atualizações das técnicas e procedimentos. $\mathrm{O}$ animal de laboratório é prioritário no campo da experimentação, e os centros de produção têm-se preocupado com seu uso e manejo. A partir desses pressupostos é imprescindível uma disciplina voltada ao estudo com animais. Assim, o objetivo desse estudo foi analisar a percepção dos discentes sobre o componente curricular Bioterismo e Biossegurança dos cursos de Bacharelado e Licenciatura em Ciências Biológicas da UFRPE. Para responder essa indagação, foi utilizado o método quantitativo e a coleta de dados foi obtida por um questionário composto por vinte perguntas aplicado a 32 alunos matriculados no primeiro período de 2018. Os resultados apontaram que houve clareza quanto ao significado e a importância da disciplina para os cursos $(90,62 \%)$ e a disciplina contribuiu para o desenvolvimento da capacidade crítica dos alunos $(84,38 \%)$. O docente cumpriu com o horário das aulas $(90,62 \%)$ e demonstrou domínio e segurança quanto aos conteúdos ministrados $(93,75 \%)$. Quanto à auto avaliação, os alunos não necessitaram de conteúdo prévio para acompanhar as aulas $(84,38 \%)$ e ficaram satisfeitos com o que aprenderam (81,25\%). Esses percentuais superiores a $80 \%$ reforçam a importância da disciplina para a matriz curricular dos cursos de Bacharelado e Licenciatura em Ciências Biológicas.
\end{abstract}

Palavras-chave: Biologia; Componente curricular; Experimentação animal. 


\section{ABSTRACT}

The continuous evolution of human knowledge, especially that of biology, promotes actions that involve animal breeding and experimentation, triggering update on techniques and procedures. The laboratory animal is a priority in the field of experimentation, and the production centers have been concerned with its use and management. Based on these assumptions, a discipline focused on animal studies is essential. Thus, the objective of the article was to analyze students' perceptions about the curricular component Animal Facility and Biosafety of the courses Bachelor and a Degree in Biological Sciences of UFRPE. To answer this question, the quantitative method was used and the data collection was obtained by a questionnaire composed of twenty questions applied to 32 students enrolled in the first period of 2018. The results showed that there was clarity as to the meaning and importance of the discipline for the courses $(90.62 \%)$ and the discipline contributed to the development of the students' critical capacity (84.38\%). The teacher complied with the class schedule $(90.62 \%)$ and showed mastery and safety regarding the contents given $(93.75 \%)$. As for self-assessment, students do not need prior assessment to follow classes $(84.38 \%)$ and are satisfied with what they say $(81.25 \%)$. These percentages above $80 \%$ reinforce the importance of the discipline to the curricular matrix of the Bachelor and Degree in Biological Sciences.

Keywords: Biology; Curricular component; Animal experimentation.

\section{INTRODUÇÃO}

O avanço da tecnologia e da "Ciência em Animais de Laboratório", está mudando paradigmas e comportamentos de pesquisadores e profissionais que utilizam animais em pesquisa (Neves; Mancini Filho \& Menezes, 2013). No Brasil, a Lei no 11.794, conhecida como Lei Arouca, regulamenta a experimentação animal em todo território nacional. Essa lei aplica-se aos animais das espécies classificadas como filo Chordata (subfilo Vertebrata); e restringem a criação e utilização de animais com finalidade científica apenas as instituições credenciadas pelo Conselho Nacional de Controle de Experimentação Animal (CONCEA), sendo a instituição de ensino e pesquisa portadora de uma Comissão de Ética no Uso de Animais (CEUA) (Brasil, 2008).

A experimentação animal é uma atividade humana com grande conteúdo ético; os problemas dessa prática surgem do conflito entre as justificativas para o uso de animais em benefício de si próprio e do homem e do ato de não causar dor e sofrimento aos mesmos (Silva \& Espírito-Santo, 2009). A maioria dos códigos internacionais que tratam das normas de pesquisa na área da saúde cita que a pesquisa desenvolvida em seres humanos deve estar fundamentada na experimentação prévia realizada em animais, em laboratórios ou em outros centros científicos (Schnaider \& Souza, 2003). Ainda de acordo com esses autores, os experimentos com animais devem ser planejados de maneira a obter o máximo de informações utilizando-se o menor número possível, alojados em ambientes adequados para evitar situações de estresses físico e mental.

Um aspecto que deve ser considerado na experimentação animal é a biossegurança, compreendida como um conjunto de ações voltadas para a prevenção, minimização ou eliminação de 
riscos inerentes às atividades em um biotério (ambiente destinado à criação e utilização de animais voltados para ensino e pesquisa) e que podem comprometer a saúde do homem, dos animais, do meio ambiente ou a qualidade dos trabalhos desenvolvidos (Silva \& Espírito-Santo, 2009). A classificação do nível de biossegurança, nos laboratórios de experimentação animal, é norteada por diversos fatores, como a patogenicidade do microrganismo infectante, virulência, via de inoculação, endemicidade, consequências epidemiológicas, disponibilidade de tratamento eficaz e de medidas profiláticas (Politi et al., 2008).

As técnicas atuais de engenharia genética e de biologia molecular permitiram a criação e produção de animais com alto padrão de qualidade genética e sanitária, possibilitando a aquisição de modelos genéticos, ecológicos e sanitariamente definidos para a realização de pesquisas (Andrade, 2002). Contudo Schnaider e Souza (2003) apontam que pesquisas estão sendo patrocinadas para desenvolver in vitro o que antes era realizado em animais vivos, entretanto o questionamento que ainda existe é até que ponto a tecnologia de substituição de animais não prejudica a evolução da ciência médica.

Uma informação que gera ainda mais questionamentos, quanto à experimentação animal, é a plena consciência de que a sensibilidade do animal é similar à humana no que se refere à dor, memória, angústia e instinto de sobrevivência devendo-se utilizar de todos os meios possíveis para minimizar a dor e o sofrimento do animal (Neves; Mancini Filho \& Menezes, 2013).

Diante dessas informações, uma disciplina de Bioterismo e Biossegurança se mostra muito importante para a matriz curricular dos cursos de Bacharelado e Licenciatura em Ciências Biológicas no que concerne a problemática envolvendo a experimentação animal. Sendo assim, o objetivo deste trabalho foi realizar um levantamento da percepção dos discentes em relação a disciplina, ao docente e uma auto avaliação que reforçam a relevância da mesma.

\section{METODOLOGIA}

O desenvolvimento do presente trabalho constituiu-se de pesquisa bibliográfica, com abordagem quantitativa, para avaliar a percepção dos discentes quanto à disciplina optativa Bioterismo e Biossegurança. Foram consultados 32 discentes dos cursos de Bacharelado e Licenciatura em Ciências Biológicas da Universidade Federal Rural de Pernambuco (UFRPE), matriculados no primeiro semestre de 2018. A coleta de dados foi realizada por meio de um questionário de múltipla escolha sobre a disciplina (Tabela 1), o professor (Tabela 2) e uma auto avaliação (Tabela 3); aplicados em sala de aula. As opções de resposta variavam em "SIM", "NÃO” e "EM PARTE". As análises utilizadas descreveram a caracterização do perfil da amostra. 
Tabela 1 - Questionário para avaliar a disciplina optativa Bioterismo e Biossegurança.

\begin{tabular}{l|l}
\hline Item & Descrição \\
\hline 1 & Ficou claro o significado e a importância da disciplina para o curso? \\
\hline 2 & Os objetivos da disciplina foram alcançados? \\
\hline 4 & Foram obedecidos os critérios de avaliação apresentados no início do semestre? \\
\hline 5 & O conteúdo da disciplina foi distribuído de forma adequada durante o semestre? \\
\hline 6 & Os métodos de avaliação conseguiram detectar se houve aprendizado? \\
\hline 8 & Os métodos de avaliação são consistentes com os objetivos propostos? \\
\hline 9 & A disciplina contribuiu para o desenvolvimento de sua capacidade crítica? \\
\hline 10 & As aulas se mostraram dinâmicas? \\
\hline
\end{tabular}

Fonte: Elaborado pelos autores (2018).

Tabela 2 - Questionário para avaliar o docente da disciplina optativa Bioterismo e Biossegurança.

\begin{tabular}{l|l}
\hline Item & Descrição \\
\hline 1 & O professor (a) cumpriu o horário das aulas? \\
\hline 2 & $\begin{array}{l}\text { O professor (a) demonstrou conhecimento atualizado, domínio e segurança aos conteúdos ministrados na } \\
\text { disciplina? }\end{array}$ \\
\hline 3 & O professor (a) utilizou metodologias adequadas ao ensino da disciplina? \\
\hline 4 & $\begin{array}{l}\text { O professor (a) utilizou instrumentos (provas, trabalhos, etc.) de avaliação compatíveis com os } \\
\text { conhecimentos, habilidades e atitudes desenvolvidas na disciplina? }\end{array}$ \\
\hline 5 & O professor mostrou-se disponível para atender aos alunos sempre que possível? \\
\hline 6 & Se você pudesse escolher, faria outra disciplina com esse professor (a) ou o indicaria para outro aluno? \\
\hline
\end{tabular}

Fonte: Elaborado pelos autores (2018).

Tabela 3 - Questionário para auto avaliação sobre disciplina optativa Bioterismo e Biossegurança.

\begin{tabular}{l|l}
\hline Item & Descrição \\
\hline 7 & Você considera necessário algum pré-requisito para acompanhar as aulas da disciplina? \\
\hline 8 & Estou satisfeito com o que aprendi na disciplina? \\
\hline 9 & Dediquei o esforço necessário à disciplina? \\
\hline 10 & Após cursar a disciplina, seu interesse pelo assunto aumentou? \\
\hline
\end{tabular}

Fonte: Elaborado pelos autores (2018).

\section{RESULTADOS E DISCUSSÃO}

Os resultados das análises dos questionários sobre a percepção dos discentes quanto à disciplina foram organizados por variável de resposta e em itens analisados em gráficos de coluna. Os percentuais superiores a $80 \%$ corresponderam aos itens 1, 2, 5 e 7 (Gráfico 1). Isso indicou que na percepção dos discentes ficou claro o significado e a importância da disciplina Bioterismo e Biossegurança para os cursos de Bacharelado e Licenciatura em Ciências Biológicas (90,62\%). Além disso, os objetivos foram alcançados permitindo o cumprimento satisfatório do programa dessa disciplina (90,32\%). Quanto à proposta pedagógica, os métodos de avaliação conseguiram detectar aprendizado (80,65\%). A disciplina também contribuiu para o desenvolvimento da capacidade crítica dos discentes $(84,38 \%)$. 
O menor percentual correspondente ao item 8 (34,38\%) indicou que a disciplina precisa se reestruturar quanto à dinâmica de aula.

Gráfico 1 - Apresentação das respostas obtidas no questionário de avaliação de percepção dos discentes matriculados nos cursos de Bacharelado e Licenciatura em Ciências Biológicas da UFRPE sobre a disciplina Bioterismo e Biossegurança.

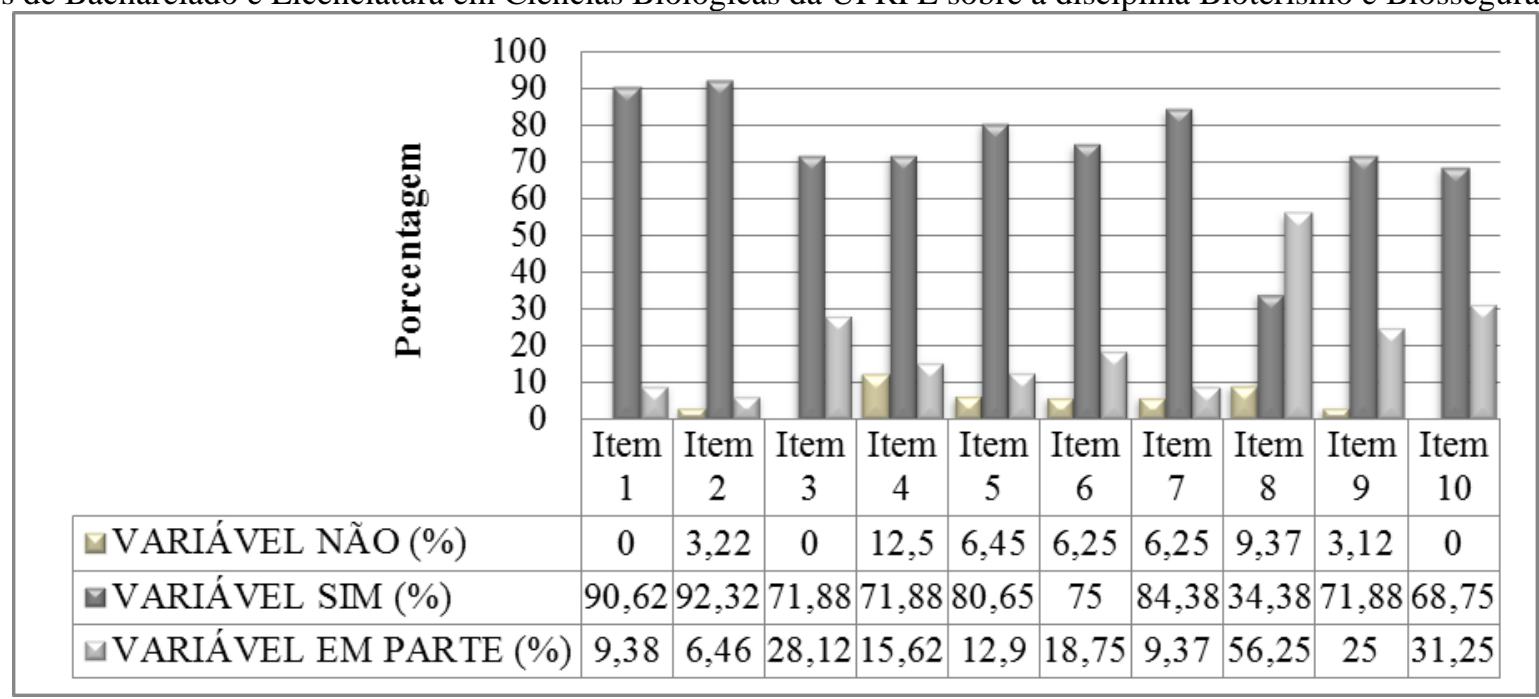

Em qualquer curso superior, o nível de ensino está relacionado a um modelo pedagógico adequado e a qualificação e atualização permanente do corpo docente, cujo intuito é proporcionar uma formação generalista, humanista, crítica e reflexiva (Lazzarin; Nakama \& Cordoni Jr, 2007).

A importância de monitorar o grau de satisfação dos acadêmicos, avaliar seus interesses e dos professores, os modelos de ensino, a matriz curricular, e a organização do curso, são maneiras de atender as recomendações requeridas pelos alunos e pela sociedade acadêmica (Vieira; Milach \& Huppes, 2008).

Analisando a percepção de discentes dos cursos da área de ciências e também de humanidades, Alencar e Fleith (2004) delimitaram 4 fatores que favorecem o desenvolvimento e a expressão da criatividade do discente, cita-se incentivo a novas ideias; clima para expressão de ideias; avaliação e metodologia de ensino e interesse pela aprendizagem do discente.

Alunos com dificuldades de aprendizagem, desinteresse pelo conteúdo ministrado, poucas oportunidades para discutir e trocar ideias com colegas de sala sobre estratégias instrucionais e elevado número de alunos constituem-se em barreiras para promoção de condições favoráveis à criatividade em sala de aula (Alenca \& Fleith, 2010).

Nenhum item demonstrou prejuízo quanto à programação da disciplina sendo registrados itens com percentuais nulos (Gráfico 1 - Variável "NÃO”). O maior percentual (12,5\% - item 4) indicou que o conteúdo da disciplina precisa ser distribuído de forma adequada durante o semestre. 
Um estudo realizado por Beck e Rausch (2014), analisou a percepção dos estudantes matriculados nos cursos de ciências contábeis, possibilitando à identificação de diferentes fatores que influenciam o processo ensino-aprendizagem, a exemplo, a motivação e a atitude com a disciplina (baseado no fator aluno); tipos de aprendizagem requeridos e a estrutura dos componentes e relações (relacionados ao fator assunto); a atitude com a matéria ensinada e a situação estimuladora ambiental (voltado ao fator professor).

No Gráfico 1, podem ser observados também os percentuais para a Variável "EM PARTE”. Os itens com valores iguais ou maiores a $25 \%$ foram 3, 8, 9 e 10 que correspondem, respectivamente, aos critérios de avaliação apresentados no início do semestre $(28,12 \%)$, dinâmica de aula $(56,25 \%)$, aulas teóricas bem estruturas e relevantes para o curso (25\%) e acessibilidade do material de apoio $(31,25 \%)$.

Para que se tornem sentenças afirmativas, esses percentuais requerem novas abordagens pedagógicas que reforcem a didática durante as aulas ministradas pelo docente. Cardoso e Bzuneck (2004) apontam que as ações do professor são importantes para socializar os acadêmicos tanto para uma motivação definida qualitativamente como em relação a estratégias de aprendizagem.

No Gráfico 2 (Variável “SIM”), os percentuais superiores a 90\% observados nos itens 1, 2, 4 indicaram que na percepção dos estudantes, o docente: cumpriu com o horário das aulas (90,7\%); demonstrou domínio quanto aos conteúdos ministrados $(93,8 \%)$ e utilizou instrumentos de avaliação compatíveis à disciplina $(93,8 \%)$. Com relação a auto avaliação (item 8), 81,3\% dos estudantes ficaram satisfeitos com o que aprenderam na disciplina. O percentual nulo (item 7) indica que não há necessidade de um pré-requisito para acompanhar as aulas.

Gráfico 2 - Apresentação das respostas obtidas no questionário de avaliação de percepção dos discentes matriculados nos cursos de Bacharelado e Licenciatura em Ciências Biológicas da UFRPE sobre a perspectiva do docente e da auto avaliação na disciplina de Bioterismo e Biossegurança.

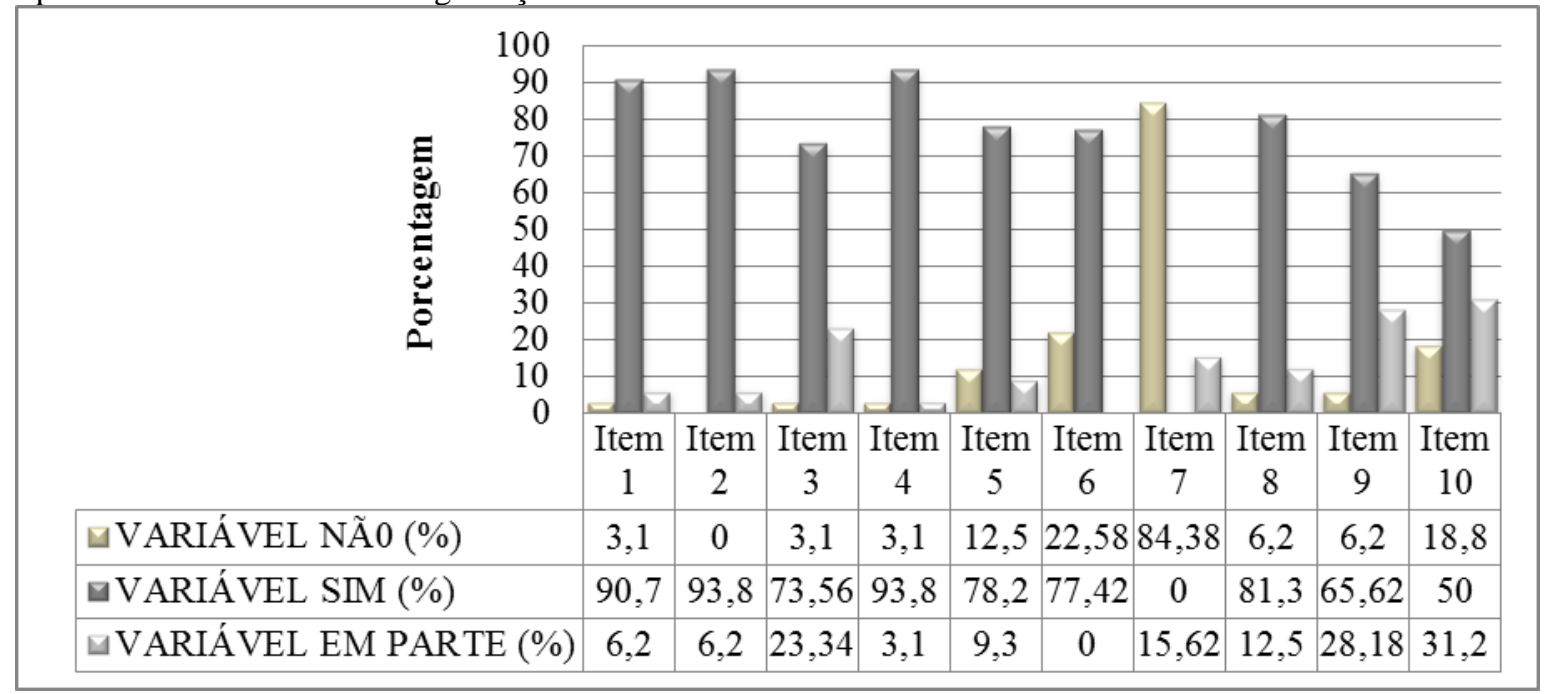


Um docente atualizado e dedicado favorece a aprendizagem dos alunos, enquanto que a falta de estímulo por parte do mesmo em se atualizar e ensinar, influência de forma contrária esse processo (Rego, 2001). Suas atitudes com a matéria ensinada, ou seja, o respeito e a dedicação, age intervindo e mediando a relação dos estudantes com o conhecimento, provocando avanços nas áreas do ensino, na relação afetivo-emocional, nas habilidades ou valores, que abrangem os aspectos do aluno/profissional requerido pelo mercado de trabalho (Beck \& Rausch, 2014).

O processo avaliativo compreende o estabelecimento dos objetivos propostos pelo professor que, na maioria das vezes, estão relacionados estreitamente a itens do conteúdo programático e a determinados intervalos (Hoffmann, 1993). Considerando essa temática, foi constatado que o professor utilizou instrumentos (provas, trabalhos, etc.) de avaliação compatíveis com os conhecimentos, habilidades e atitudes desenvolvidas na disciplina (item 4).

Os percentuais quanto a Variável "NÃO" para os itens analisados no Gráfico 2 indicam que para $84,38 \%$ dos discentes não há necessidade de algum pré-requisito para acompanhar as aulas da disciplina (item 7).

Os resultados obtidos em relação a Variável "EM PARTE” (Gráfico 2 ) mostram que os maiores percentuais para os itens 3, 9 e 10 correspondem, respectivamente, a utilização de metodologias adequadas $(23,34 \%)$, se os alunos dedicaram algum esforço à disciplina $(28,18 \%)$ e se após cursar, o interesse pelo assunto aumentou $(31,2 \%)$.

Os valores abaixo de $23 \%$, não foram representativos para caracterizar uma resposta ou efeito relevante aos itens avaliados, mas servem para mostra o que pode e deve ser ajustado ou revisto.

Trabalhos envolvendo a percepção dos discentes foram realizados por diversos autores com diferentes finalidades. A pesquisa de Oliveira e Wechsler (2002) buscou analisar algumas das variáveis que afetam o processo de ensino-aprendizagem, a partir da percepção dos discentes dos cursos de licenciatura e de professores da rede estadual, concluindo que é necessário dar mais ênfase ao processo de criatividade nos cursos de licenciatura.

Borges et al. (2013) tiveram como objetivo traçar um perfil do panorama geral do ensino da medicina veterinária em relação a questões de bem-estar e dor animal, concluindo que o ensino brasileiro enfatiza a esfera física do bem-estar animal, sendo importante o enriquecimento em relação às esferas comportamental, psicológica e ao ensino da dor.

Pádua Júnior et al. (2014), entrevistando alunos e professores do curso de administração, verificaram que as inovações no ensino passam pela maior autonomia do aluno e por projetos multidisciplinares, os quais possibilitam uma visão mais geral, não se restringindo à disciplina. 


\section{CONCLUSÕES}

Os resultados apresentados quanto à disciplina de Bioterismo e Biossegurança mostraram-se relevantes para a matriz curricular dos cursos de Bacharelado e Licenciatura em Ciências Biológicas da UFRPE, pois revela a necessidade de qualquer instituição de ensino superior em monitorar a percepção dos alunos em relação à determinada disciplina para garantir a melhoria de ensino.

A atitude do docente com a matéria ensinada e o relacionamento com os estudantes proporcionam avanços na área do conhecimento, assim como aumenta o interesse pela disciplina. Um professor atualizado e dedicado favorece a aprendizagem e suas ações positivas exercem efeitos motivadores sobre seus alunos. 


\section{REFERÊNCIAS BIBLIOGRÁFICAS}

Alencar, E. M. L. S. de, \& Fleith, D. S. (2004). Inventário de práticas docentes que favorecem a criatividade no ensino superior. Psicologia: Reflexão e Crítica, 17(1), 105-110.

Alencar, E. M. L. S. de, \& Fleith, D. S. (2010). Criatividade na educação superior: fatores inibidores. Avaliação, 15(2), 201-219.

Andrade, A. (2002). Biossegurança em Biotérios. In: A. ANDRADE; S. C. PINTO; R. S. OLIVEIRA, (Ed.), (pp. 381-386). Rio de Janeiro: Editora Fiocuz.

Beck, F., \& Rausch, R. B. (2014). Fatores que influenciam o processo ensino-aprendizagem na percepção de discentes do curso de ciências contábeis. Contabilidade vista \& revista, 25(2), 38-58.

Borges, T. D.; Sans, E. C. O.; Braga, J. S.; Machado, M. F., \& Molento, C. F. M. (2013). Ensino de bem-estar e dor animal em cursos de medicina veterinária no Brasil. Arquivo Brasileiro Medicina Veterinária e Zootecnia, 65(1), 29-36.

Brasil. Lei n ${ }^{\circ} 11.794$, de 8 de outubro de 2008. Estabelece os procedimentos para o uso científico de animais. Diário Oficial da União, Brasília, DF, 09 out. 2008.

Cardoso, L. R., \& Bzuneck, J. A. (2004). Motivação no ensino superior: metas de realização e estratégias de aprendizagem. Psicologia Escolar e Educacional, 8(2), 145-155.

Hoffmann, J. M. L. (1993). Avaliação: Mito \& desafio. Uma perspectiva construtivista. Porto Alegre: Educação e Realidade.

Lazzarin, H. C.; Nakama, L., \& Cordoni Júnior, L. (2007). O papel do professor na percepção dos alunos de Odontologia. Saúde e Sociedade, 16(1), 90-101.

Neves, S. M. P.; MancinI Filho, J., \& Menezes, E. W. de. (2013). Manual de Cuidados e Procedimentos com Animais de Laboratório do Biotério de Produção e Experimentação da FCF-IQ/USP. São Paulo: Universidade de São Paulo, Faculdade de Ciências Farmacêuticas, Instituto de Química (FCFIQ/USP), 234 p.

Oliveira, E. T. A., \& Wechsler, S. M. (2002). Variáveis que afetam a aprendizagem: percepção de alunos de licenciatura e professores. Psicologia escolar e educacional, 6(2), 133-139.

Pádua Júnior, F. P de; Castilho Filho, J. P. de; Steiner Neto, P. J., \& Akel Sobrinho, Z. (2014). Avaliação da percepção de discentes e docentes sobre novas tecnologias de ensino em cursos de graduação em administração. Administração: ensino e pesquisa, 15(2), 295-321.

Politi, F. A. S.; Majerowicz, J.; Cardoso, T. A. O.; Pietro, R. C. L. R., \& Salgado, H.R.N. (2008). Caracterização de biotérios, legislação e padrões de biossegurança. Revista de Ciências Farmacêuticas Básica e Aplicada, 29(1),17-28.

Rego, A. (2001). O bom cidadão docente universitário na senda da qualidade no ensino superior. Educação \& Sociedade, Campinas, 22(75), 174-199.

Schnaider, T. B., \& Souza, C. de (2003). Aspectos Éticos da Experimentação Animal. Revista Brasileira de Anestesiologia, 53(2), 278-285. 
Silva, M. L. P. C. da, \& Espírito-Santo, N. B. do. (2009). Bioterismo - Ciência e Biotecnologia. Revista Interdisciplinar de Estudos Experimentais, 1(3), 131-139.

Vieira, K. M.; Milach, F. T., \& Huppes, D. (2008). Equações estruturais aplicadas à satisfação dos alunos: um estudo no curso de ciências contábeis. Revista Contabilidade \& Finanças-USP, 19(48), 6576. 